\title{
Evaluación de la Respuesta Inflamatoria en Ratas Wistar a TheraCalTM LC Implantado Vía Subcutánea
}

\author{
Evaluation of the Inflammatory Response in Wistar \\ Rats to TheraCalTM LC Implanted Subcutaneously
}

\begin{abstract}
Paulina Alejandra Flores de los Rios ${ }^{1}$; Francela Valeria Borja Hernández²; Velia Muñoz Alanis²; Adolfo Soto Domínguez ${ }^{3}$; Claudia Alicia Meléndez Wong²; Rubén García Garza ${ }^{1}$; Joel Jiménez Villarreal ${ }^{4}$;Nadia Denys Betancourt Martínez; ${ }^{5}$ Daniel Almaraz Célis ${ }^{6}$ \& Javier Morán Martínez ${ }^{5,6}$
\end{abstract}

FLORES DE LOS RIOS,P.A.; BORJA, H. F. V.; MUÑOZ,A.V.; SOTO, D.A.; MELÉNDEZ,W.C.A.; GARCÍA, G. R.; JIMÉNEZ, V. J.; BETANCOURT, M. N. D.; ALMARAZ, C. D. \& MORÁN, M. J. Evaluación de la respuesta inflamatoria en ratas wistar a TheraCalTM LC implantado vía subcutánea. Int. J. Morphol., 37(2):685-689, 2019.

RESUMEN: El Theracal TM LC es un cemento silicato de calcio (Ca) modificado con resina (SMCR) que ha demostrado ser un material ideal para el tratamiento dentino-pulpar por su alta tasa de formación de calcio. Los biomateriales por su contenido de Ca tienden a tener un aumento en su biodisponibilidad, estimulando la formación del puente dentario atreves de las células involucradas en la formación de tejidos mineralizados, promoviendo la diferenciación de fibroblastos en odontoblastos y aumentando la actividad de la enzima pirofostasa responsable en la mineralización de la dentina. El presente estudio con el objetivo de evaluar la respuesta inflamatoria a Theracal TM LC subcutáneamente en ratas Wistar. Fueron usados seis ratas cepa Wistar en las cuales se realizaron cuatro bolsillos quirúrgicos subcutáneos. Cada uno de estos bolsillos se determinó como cuadrante distinto, conteniendo los siguientes implantes: 1 Theracal TM LC en tubo polietileno, 2 tubo de polietileno, 3 Theracal TM LC directo y 4 como control. Las muestras histológicas se procesaron y se evaluaron distintos tipos celulares mediante conteo a microscopio de luz a 100X utilizando las tinciones H\&E y AT pH 2.3. Los resultados mostraron que existen diferencias significativas en todos los tipos celulares observados durante los diferentes tiempos de exposición. Las diferencias en los tipos celulares observados podrían ser debido al tiempo de exposición al Theracal TM LC, al tubo polietileno y a ambos. El tejido evaluado del implante del tubo polietileno y al tubo polietileno con Theracal TM LC, presentan mayor respuesta inflamatoria, a diferencia en el tejido implantado con Theracal TM LC directamente.

PALABRAS CLAVE: Respuesta inflamatoria; Ratas Wistar; Calcio.

\section{INTRODUCCIÓN}

En la actualidad existen biomateriales, biocerámicos y cementos, los cuales son de gran utilidad en odontología, principalmente cuando se usan en el ligamento periodontal y el hueso alveolar, fracturas, perforaciones u obturaciones retrógradas (Broon et al., 2016), los componentes principales de estos biomateriales son a base de calcio $(\mathrm{Ca})$; fosfato monobásico, silicatos de calcio, hidróxido de calcio, óxido de zirconio y agentes de relleno y espesantes (De-Deus et al., 2009; Broon et al.). Cuyos objetivos principales son mantener en posición la restauración y evitar la filtración bacteriana y la microfiltración entre el cemento y el diente de agentes externos (Costa et al., 2000; Dickens et al., 2010).
El TheraCal TM LC, es un cemento silicato de calcio modificado con resina (SMCR), fotocurable o fotopolimerizable, perteneciente a los MTA (Agregado de Mineral Trióxido), su función principal es formar una barrera protectora del complejo dentino- pulpar. Su formulación consta de $45 \%$ de partículas de silicato tricálcico (cemento Portland tipo III), $10 \%$ de estroncio que lo provee de radiopacidad, $5 \%$ de sílica pirogénica que le otorga características hidrofílicas y $45 \%$ de contenido de resina, así como un $\mathrm{pH}$ alcalino. Por su contenido de $\mathrm{Ca}$ en los biomateriales generan un aumento de biodisponibilidad de esté, estimulando la formación del puente dentario atreves de las células

\footnotetext{
${ }^{1}$ Departamento de Histología, Facultad de Medicina Unidad Torreón, Universidad Autónoma de Coahuila, Torreón, Coahuila, México.

${ }^{2}$ Clínica de Odontología Infantil, Facultad de Odontología Unidad Torreón, Universidad Autónoma de Coahuila, Torreón, Coahuila, México.

${ }^{3}$ Departamento de Histología, Facultad de Medicina, Universidad Autónoma de Nuevo León. Monterrey, Nuevo León, México.

${ }^{4}$ Departamento de Investigación, Escuela de Medicina Unidad Norte, Universidad Autónoma de Coahuila, Piedras Negras, Coahuila, México.

${ }^{5}$ Departamento de Biología Celular y Ultraestructura, Centro de Investigación Biomédica, Facultad de Medicina Unidad Torreón, Universidad Autónoma de Coahuila, Torreón, Coahuila, México.

${ }^{6}$ Coordinación de Investigación, Escuela de Medicina, Universidad Autónoma de Durango, Campus Gómez Palacio. Gómez Palacio, Durango, México.
} 
involucradas en la formación de tejidos mineralizados, promoviendo la diferenciación de fibroblastos en odontoblastos y aumentando la actividad de la enzima pirofostasa responsable en la mineralización de la dentina (Cintra et al., 2010; Gandolfi et al., 2012; Cedillo Valencia \& Cedillo Félix, 2013). Sin embargo, a pesar de que los biomateriales mantienen un nivel de biocompatibilidad aceptable por las características de sus componentes, se busca la posible respuesta biológica y los efectos adversos de los materiales de uso odontológico desde la toxicidad, inflamación, alergia y mutabilidad (Rojas, 1995; Villegas et al., 2008).

Las pruebas de biocompatibilidad son pruebas iniciales como ensayos de citotoxicidad, respuesta inflamatoria y carcinogénesis, además las pruebas secundarias permiten medir los niveles de reacciones inflamatorias o de respuestas inmunitarias frente al material (Rojas; Berrios Quina \& Porto Neto, 2004; Villegas et al.). Diversos estudios realizados en dentición de animales, implantes subcutáneos y cultivos celulares, han sido utilizados para la evaluación sobre la biocompatibilidad de materiales usados en odontología (Cintra et al.). La prueba más utilizada es la implantación a través del tejido subcutáneo en ratas, el cual se emplea para evaluar diversos materiales dentales de uso en terapia pulpar u obturación radicular (Villegas et al.). Sin embargo, existen pocos reportes científicos que reporten el uso específico del TheraCal TM LC y su biocompatibilidad con el tejido subdérmico y el organismo.

El presente tiene como objetivo determinar la respuesta inflamatoria en ratas Wistar implantadas son TheraCal TM LC en tubos de polietileno en tejido subdérmico.

\section{MATERIAL Y MÉTODO}

Se diseñó un estudio experimental, comparativo, prospectivo longitudinal en un modelo animal (rata Wistar), se realizó en el Bioterio de la Facultad de Medicina, Unidad Torreón, Universidad Autónoma de Coahuila, Coahuila, México. Apegándose a la Norma Oficial Mexicana (NOM062-ZOO- 1999), además la investigación fue aprobada por el comité de Bioética de la Facultad (No. CONBIETICA07CEI00320131015).

Modelos animales. Se utilizaron seis ratas Wistar entre machos y hembras con un rango de peso de 200 a $300 \mathrm{~g}$, a todos los modelos animales se les mantenía limpieza constante, bajo condiciones de temperatura controlada de 25-26 ${ }^{\circ} \mathrm{C}$, con humedad relativa de $30-70 \%$ y con ciclos de luz y oscuridad de $12 \mathrm{~h}$. Todos los animales fueron alimentados con croquetas para ratas (HARLAN $®)$ y agua ad libitum.
El estudio fue realizado en el bioterio certificado por la Secretaría de Agricultura, Ganadería, Desarrollo Rural, Pesca y Alimentación (SAGARPA) y Servicio Nacional de Sanidad, Inocuidad y Calidad Agroalimentaria (SENASICA) de la Facultad de Medicina de la Universidad autónoma de Coahuila Unidad Torreón, México; con número de certificación: AUT-B-C-0318-42. Todos los procedimientos fueron supervisados por un veterinario certificado por SAGARPA y SENASICA (No. 4807528).

Procedimiento. Para la implantación del tubo polietileno y del TheraCal TM LC (Bisco Inc., Schaumburg, IL, USA), los animales fueron anestesiados con clorhidrato de ketamina $500 \mathrm{mg} / 10 \mathrm{~mL}$ y diazepam $2 \mathrm{mg} / 1 \mathrm{~mL}$ en dosificación de 2 $\mathrm{mL} / 100 \mathrm{~g}$ y se permitió un tiempo de anestesia de aproximadamente $20 \mathrm{~min}$. El dorso fue marcado en cuatro cuadrantes y se rasuraron hasta dejar la epidermis libre de pelaje y fue desinfectada con iodo en alcohol al $5 \%$. Bajo condiciones asépticas se realizaron tres incisiones de aproximadamente $12 \mathrm{~mm}$ de largo por $20 \mathrm{~mm}$ de profundidad realizada mediante una tijera de punta roma permitiendo abrir el tejido subcutáneo de manera lateral para obtener dos ventanas quirúrgicas una a cada lado de la incisión sin que se producción de hemorragia, además, se realizaron dos incisiones con cuatro bolsillos quirúrgicos para los materiales.

Implantación de los materiales. Cuadrante uno; se implantó un tubo de polietileno de $5 \mathrm{~mm}$ de longitud por 1,3 $\mathrm{mm}$ de diámetro relleno de TheraCal TM LC.Cuadrante dos; tubo de polietileno de $5 \mathrm{~mm}$ de longitud por 1,3 $\mathrm{mm}$ de diámetro Cuadrante tres; se colocó $8 \mathrm{~mm}$ de TheraCal TM LC en el tejido directamente. Cuadrante cuatro; Considerado control.

La colocación de los tubos se realizó con una pinza curva y en un sentido paralelo a la incisión y con el extremo abierto en dirección cefálica quedando en contacto con tejido conjuntivo. Posteriormente se fotopolimerizó TheraCal TM LC de los cuadrantes uno y tres con una lámpara de fotopolimerización a $6 \mathrm{~mm}$ de distancia por 20 segundos. Después de la implantación las incisiones fueron suturadas con Vicril de 3 ceros y se limpiaron las zonas de incisión. Después de 7, 15 y 30 días posteriores a la implantación, se obtuvieron biopsias excisionales de cada cuadrante y se fijaron con formol al $10 \%$, las cuales fueron procesadas para su evaluación histopatológica.

Evaluación histopatológica. Se realizo la técnica histopatológica convencional de inclusión en parafina y se realizaron cortes histológicos de 5 a 7 micras y se tiñeron con hematoxilina eosina (H\&E) y Azul de Toluidina (AT) pH 2,3. los cortes histopatológicos que fueron teñidos con H\&E fueron observados en un microscopio de luz de campo claro a $100 \mathrm{X}$, evaluando ocho campos, en los cuales se 
FLORES DE LOS RIOS, P. A.; BORJA, H. F. V.; MUÑOZ, A. V.; SOTO, D. A.; MELÉNDEZ, W. C. A.; GARCÍA, G. R.; JIMÉNEZ, V. J.; BETANCOURT, M. N. D.; ALMARAZ, C. D. \& MORÁN, M. J. Evaluación de la respuesta inflamatoria en ratas wistar a TheraCalTM LC implantado vía subcutánea. Int. J. Morphol., 37(2):685-689, 2019.

realizó un conteo de células polimorfonucleares, neutrófilos, macrófagos, linfocitos y células plasmáticas por campo. Los cortes teñidos con AT pH 2,3 se observaron con el microscopio de campo claro a 100X, evaluando ocho campos en los cuales se realizó un conteo de células cebadas.

Análisis estadístico. Todos los resultados muestran media y desviación estándar siguiendo las caracterices de las variables fueron analizadas mediante la prueba estadística de $\mathrm{t}$ de Student considerando estadísticamente significativos valores de $\mathrm{p}<0,05$. El paquete estadístico SPSS versión 21 fue utilizado para este análisis.
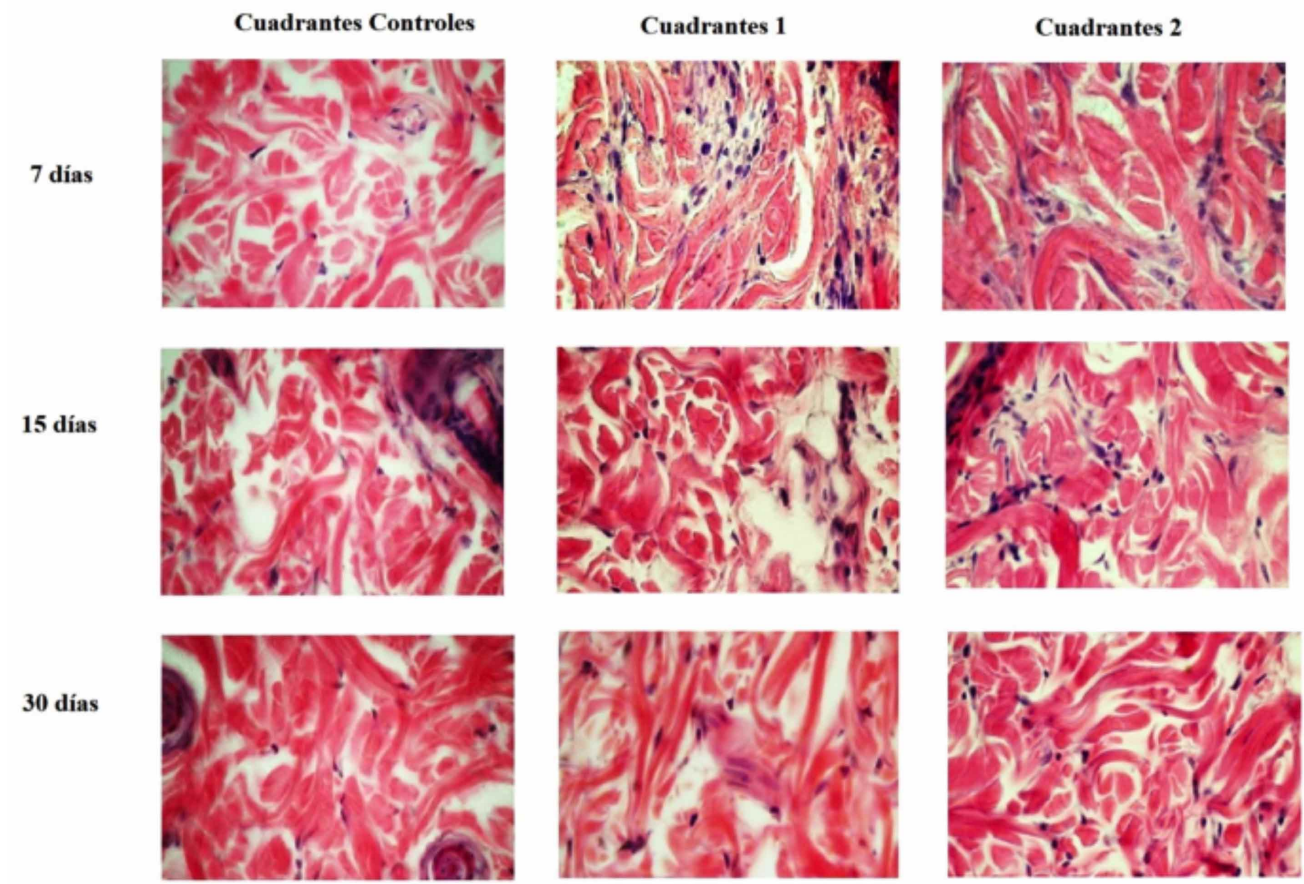

En la evaluación de la respuesta inflamatoria en los tres cuadrantes tratados se observó en la evaluación del séptimo día un incremento significativo en el conteo de células polimorfonucleares, linfocitos, macrófagos y células plasmáticas todos respecto al cuadrante control. La evaluación del decimoquinto día no existió diferencia de en las líneas celulares evaluadas. En la evaluación del día 30 se mostró un incremento en el número de polimorfonucleares, linfocitos, macrófagos, células plasmáticas y células ceba-
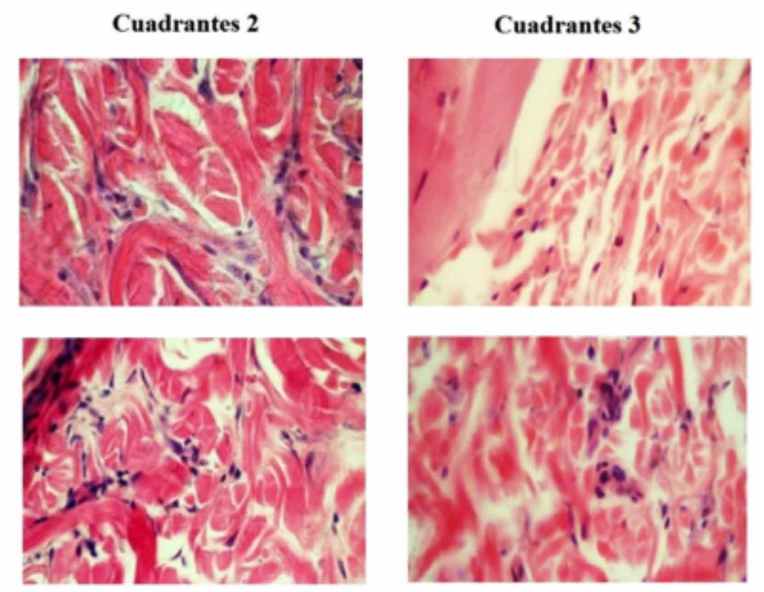

Fig. 1. Tinción con H\&E. Diferencia del infiltrado inflamatorio en los cuadrantes vs los controles en los 7, 15 y 30 días respectivamente.

Tabla I. Conteo histológico de tejido subdérmico con las tinciones de H\&E y de AT pH 2.3 a los 7, 15 y 30 días posteriores a la implantación.

\begin{tabular}{|c|c|c|c|c|c|}
\hline Cuadrante & $\mathrm{C}$, polimorfonucleares & Linfocitos & Macrófagos & $\mathrm{C}$, plasmáticas & $\mathrm{C}$, cebadas \\
\hline \multicolumn{6}{|l|}{ Día 7} \\
\hline Tubo + Theracal ${ }^{\mathrm{TM}} \mathrm{LC}$ & $1,06 \pm 0,27 *$ & $18,19 \pm 2,56^{*}$ & $7,31 \pm 0,27 *$ & $3,19 \pm 1,15^{*}$ & $1,31 \pm 0,80$ \\
\hline Tubo vacío & $1,88 \pm 0,71 *$ & $21,06 \pm 8,75^{*}$ & $7,94 \pm 2,56^{*}$ & $2,81 \pm 0,27 *$ & $1,44 \pm 0,44$ \\
\hline Theracal ${ }^{\mathrm{TM}} \mathrm{LC}$ & $0,50 \pm 0,53$ & $13,94 \pm 0,80^{*}$ & $4,38 \pm 1,41^{*}$ & $1,75 \pm 0,18^{*}$ & $3,00 \pm 2,30$ \\
\hline Control & 0,00 & $2,06 \pm 1,86$ & $0,13 \pm 0,00$ & $0,25 \pm 0,18$ & $1,25 \pm 0,18$ \\
\hline \multicolumn{6}{|l|}{ Día 15} \\
\hline Tubo + Theracal ${ }^{\mathrm{TM}} \mathrm{LC}$ & $0,88 \pm 1,06$ & $10,85 \pm 5,87$ & $3,68 \pm 2,03$ & $2,10 \pm 1,31$ & $1,85 \pm 0,79$ \\
\hline Tubo vacío & $0,88 \pm 1,06$ & $12,00 \pm 6,01$ & $3,85 \pm 2,01$ & $2,08 \pm 1,31$ & $2,10 \pm 0,70$ \\
\hline Theracal ${ }^{\mathrm{TM}} \mathrm{LC}$ & $0,70 \pm 1,08$ & $10,18 \pm 5,67$ & $3,23 \pm 1,56$ & $2,35 \pm 1,34$ & $2,00 \pm 0,64$ \\
\hline Control & $0,75 \pm 1,07$ & $7,15 \pm 5,82$ & $2,63 \pm 2,10$ & $1,95 \pm 1,72$ & $1,90 \pm 0,74$ \\
\hline \multicolumn{6}{|l|}{ Día 30} \\
\hline Tubo + theracal ${ }^{\mathrm{TM}} \mathrm{LC}$ & 0,00 & $9,25 \pm 3,36^{*}$ & $4,13 \pm 0,35^{*}$ & $3,63 \pm 1,94 *$ & $3,50 \pm 2,12$ \\
\hline Tubo vacío & $1,44 \pm 2,03 *$ & $8,88 \pm 3,18^{*}$ & $8,38 \pm 4,60^{*}$ & $2,44 \pm 0,27 *$ & $5,77 \pm 2,68^{*}$ \\
\hline Theracal ${ }^{\mathrm{TM}} \mathrm{LC}$ & $0,06 \pm 0,09 *$ & $11,00 \pm 3,36^{*}$ & $12,81 \pm 2,56^{*}$ & $1,56 \pm 0,44^{*}$ & $2,22 \pm 1,77$ \\
\hline Control & 0,00 & $1,31 \pm 0,44$ & $0,31 \pm 0,09$ & $0,25 \pm 0,00$ & $2,39 \pm 0,37$ \\
\hline
\end{tabular}

*p<0.05 Estadísticamente significativo con respecto al grupo control. 
das (Tabla I). En el cuadrante control se observó la dermis y tejido subcutáneo de características histológicas normales con presencia de escaso infiltrado inflamatorio.

Las observaciones a 40X con la tinción H\&E muestran la diferencia del infiltrado inflamatorio en los diferentes cuadrantes con respecto a lo observado en los controles a $\operatorname{los} 7,15$ y 30 días respectivamente (Fig. 1).

\section{DISCUSIÓN}

La aparición del infiltrado inflamatorio observado a los 7 días de nuestro estudio experimental concuerda con lo reportado por Souza et al. (2006) quienes demostraron que posterior al implante de liners de ionómeros de vidrio se presentaba una respuesta inflamatoria moderada observando neutrófilos, macrófagos y linfocitos. Otro estudio utilizando tubos de polietileno con MTA por Cintra et al. en huecos alveolares de ratas y también en bolsillos quirúrgicos de tejido conectivo subepitelial, reportó pocas células inflamatorias a los 30 días de exposición, a diferencia de lo observado en nuestro estudio, en donde a los 30 días el infiltrado inflamatorio aun persistía, con predominio linfocítico y de macrófagos. Aunque el incremento de los distintos tipos celulares se presentó en todos los cuadrantes a los 7 días, el observado para el cuadrante implantado únicamente con TheraCal TM LC (linfocitos 13,94 $\pm 0,80$, macrófagos $4,38 \pm 1,41$ y células plasmáticas $1,75 \pm 0,18$ ), fue considerablemente menor al observado en el grupo con implante que contuvo el TheraCal TM LC en tubos de polietileno y el grupo implantado con el tubo polimerizado, los cuales presentaron valores en tipos celulares de células polimorfonucleares $1,06 \pm 0,27$, linfocitos $18,19 \pm 2,56$, macrófagos 7,3 $\pm 0,27$ y células plasmáticas $3,19 \pm 1,15$ y, células polimorfonucleares $1.88 \pm 0.71$, linfocitos $21,06 \pm 8,75$, macrófagos $(7,94 \pm 2,56)$ y células plasmáticas $2,81 \pm 0,27$ respectivamente.

En nuestro estudio observamos un predominio de polimorfonucleares a los 7 días y, de linfocitos y macrófagos a los 30 días, lo que concuerda con lo reportado en la literatura por Kumar et al. (2003) quienes describieron que en el infiltrado agudo se promueve el cambio vascular y el cambio en el flujo sanguíneo y posteriormente, se presentan los cambios celulares con predominio de neutrófilos con el objetivo de fagocitar el agente agresor $y$, por su parte, el infiltrado crónico (por semanas, meses o años) con predominio de linfocitos y macrófagos, se activa debido a que el proceso agudo no es capaz de eliminar el agente agresor o bien, se ve impedido el proceso de respiración celular por diversos agentes, siendo probablemente en nuestro trabajo, debi- do al tubo de polietileno. Por otro lado, las células cebadas no mostraron incremento significativo en la mayoría de los grupos de estudio, lo que difiere con lo reportado por Campos Quintana et al. (2003) quien describió un incremento en este tipo celular a los 15, 30 y 45 días de exposición a Cemento Portland con tubo polimerizados, atribuyéndole a este hallazgo una reacción alérgica al plástico.

En este estudio se describe por primera vez la presencia de infiltrado inflamatorio ocasionado por el implante de TheraCal TM LC vía subcutánea en ratas Wistar, siendo éste similar al descrito para otros compuestos relacionados al TheraCal TM LC de uso odontológico (Campos Quintana et al.; Hebling et al., 2009; Cintra et al.). Además, el presente se distingue por arrojar datos cuantitativos de tipos celulares y no solo cualitativos o descriptivos como en la mayoría de los artículos relacionados.

En nuestro estudio observamos una reacción inflamatoria en todos los grupos experimentales, sin embargo, la tendencia es mayor en los grupos con injerto de tubo polimerizado y TheraCal TM LC en el tubo polietileno, por lo que podemos concluir que la aplicación únicamente del tubo es suficiente para producir una reacción inflamatoria más acentuada que al injertar directamente el TheraCal TM LC. A los 30 días de nuestro estudio, aún se observa una respuesta inflamatoria con el uso del TheraCal TM LC lo que concuerda con otros estudios relacionados (Campos Quintana et al.; Cintra et al.), donde también determinaron que, al mismo tiempo de exposición, la respuesta es escasa.

En conclusión, el área con implantación del tubo polietileno y el tubo polietileno con TheraCal TM LC presentan mayor respuesta inflamatoria que el TheraCal TM LC aplicado de manera directa. No se observaron efectos adversos en áreas distantes al sitio de implante, por lo que deducimos que el TheraCal TM LC no tiene efectos nocivos a distancia. Nuestros resultados en conjunto con los de otros estudios, parecen indicar que este material es útil en el tratamiento de complicaciones odontológicas; sin embargo, consideramos necesario realizar la evaluación del TheraCal TM LC en pacientes para confirmar lo anterior.

FLORES DE LOS RIOS, P. A.; BORJA, H. F. V.; MUÑOZ,A. V.; SOTO, D. A.; MELÉNDEZ, W. C. A.; GARCÍA, G. R.; JIMÉNEZ, V. J.; BETANCOURT, M. N. D.; ALMARAZ, C. D. \& MORÁN, M. J. Evaluation of the inflammatory response in Wistar rats to TheraCalTM LC implanted subcutaneously. Int. J. Morphol., 37(2):685-689, 2019.

SUMMARY: TheraCalTM LC is a resin-modified calcium silicate (Ca) resin (SMCR) that has proven to be an ideal material for dentin-pulp treatment due to its high rate of calcium formation. 
Biomaterials due to their Ca content tend to have an increase in their bioavailability, stimulating the formation of the dental bridge through the cells involved in the formation of mineralized tissues, promoting the differentiation of fibroblasts in odontoblasts and increasing the activity of the pyrophosphate enzyme responsible in dentin mineralization. The present study aimed to evaluate the inflammatory response to TheracalTM LC subcutaneously in Wistar rats. Six Wistar strain rats were used in which four subcutaneous surgical pockets were made. Each of these pockets was determined as a different quadrant, containing the following implants: 1 TheracalTM LC in polyethylene tube, 2 polyethylene tubes, 3 TheracalTM LC direct and 4 as control. The histological samples were processed, and different cell types were evaluated by light microscopy at $100 \mathrm{X}$ using the H\&E and AT pH 2.3 stains. The results showed that there are significant differences in all cell types observed during the different exposure times. The differences in the cell types observed could be due to the exposure time to TheracalTM LC, to the polyethylene tube and to both. The evaluated tissue of the polyethylene tube implant and the polyethylene tube with TheracalTM LC present a greater inflammatory response, unlike in the tissue implanted with TheracalTM LC directly.

Calcium.

KEY WORDS: Inflammatory response; Wistar rats;

\section{REFERENCIAS BIBLIOGÁFICAS}

Berrios Quina, E. J. \& Porto Neto, S. T. Respuesta pulpar frente a diferentes agentes cementales. Rev.Estomatol. Herediana, 14(1-2):84-8, 2004.

Broon, J. N.; Martínez Martínez, P. A.; Ramírez Muñiz, G. L.; Tinajero Morales, C.; Lagunas Ángeles, H. L. \& Bramante, C. M. Respuesta inflamatoria de Bioceramic a la implantación de tubos de dentina en tejido subcutáneo de ratas. Rev. Odontol. Mex., 20(3):174-8, 2016.

Campos Quintana, I.; Llamosas Hernández, E.; \& Morales de la Luz, R. Evaluación de la biocompatibilidad del cemento Portland implantado en tejido conectivo subepitelial de ratas. Rev.Asoc.Den.Mex., 60(2):45$51,2003$.

Cedillo Valencia, J. J. \& Cedillo Félix, J. E. Protocolo clínico actual para restauraciones profundas. Rev. Asoc. Dent. Mex., 70(5):263-75, 2013.

Cintra, L. T.; Bernabé, P. F.; de Moraes, I. G.; Gomes-Filho, J. E.; Okamoto, T.; Consolaro, A. \& Pinheiro, T. N. Evaluation of subcutaneous and alveolar implantation surgical sites in the study of the biological properties of root-end filling endodontic materials. J. Appl. Oral Sci., 18(1):75-82, 2010

Costa, C. A.; Teixeira, H. M.; do Nascimento, A. B. \& Hebling, J. Biocompatibility of two current adhesive resins. J. Endod., 26(9):5126, 2000.

De-Deus, G.; Canabarro, A.; Alves, G.; Linhares, A.; Senne, M. I. \& Granjeiro, J. M. Optimal cytocompatibility of a bioceramic nanoparticulate cement in primary human mesenchymal cells. J. Endod., 35(10):1387-90, 2009.

Dickens, S. H.; Flaim, G. M.; Schumacher, G. E.; Eichmiller, F. C.; Schafer, D. R. \& Rutherford, R. B. Preclinical effectiveness of a novel pulp capping material. J. Endod., 36(7):1222-5, 2010.

Gandolfi, M. G.; Siboni, F. \& Prati, C. Chemical-physical properties of TheraCal, a novel light-curable MTA-like material for pulp capping. Int. Endod. J., 45(6):571-9, 2012.

Hebling, J.; Lessa, F. C.; Nogueira, I.; Carvalho, R. M. \& Costa, C. A. Cytotoxicity of resin-based light-cured liners. Am.J. Dent., 22(3):13742,2009
Kumar, V.; Cotran, R. S. \& Robbins, S. L. Robbins Basic Pathology. $18^{\mathrm{a}}$ ed. Philadelphia, Saunders, 2003.

Rojas, W. Inmunología. 11 a ed. Medellín, Corporación para Investigaciones Biológicas, 1995. pp.47-66.

Souza, P. P.; Aranha, A. M.; Hebling, J.; Giro, E. M. \& Costa, C. A. In vitro cytotoxicity and in vivo biocompatibility of contemporary resinmodified glass-ionomer cements. Dent. Mater., 22(9):838-44, 2006.

Villegas, A. M.; Naranjo, E. \& Gómez, D. M. Pruebas de biocompatibilidad de los materiales de uso odontológico: Revisión de la literatura. Rev. Estomat., 16(2):38-44, 2008.

\author{
Dirección para correspondencia: \\ Javier Morán Martínez \\ Gregoria A. Garcia, 198 sur \\ Departamento de Biología Celular y Ultraestructura \\ Centro de Investigación Biomédica \\ Facultad de Medicina Unidad Torreón \\ Universidad Autónoma de Coahuila \\ Torreón, Coahuila, C.P. 27000. \\ MÉXICO
}

Email: javmoran@yahoo.com

Recibido: 26-07-2018

Aceptado: 21-12-2018 